\title{
Naloxone-Induced Non-Cardiogenic Pulmonary Edema: A Case Report
}

\author{
Nasheena Jiwa $^{1} \cdot$ Himesh Sheth ${ }^{1} \cdot$ Richard Silverman $^{1,2}$
}

Published online: 10 May 2018

(c) The Author(s) 2018

\begin{abstract}
A 22-year-old man was admitted for an elective right-shoulder open subacromial decompression and distal clavicle excision. He received a single intravenous dose of fentanyl $50 \mu \mathrm{g}$ for anesthesia. His procedure was completed without intra-operative complications; however, he developed post-operative respiratory depression in the setting of narcotic administration. He was given naloxone $0.2 \mathrm{mg}$ intravenously once to reverse this effect, which subsequently led to acute hypoxic respiratory failure secondary to pulmonary edema shortly after administration of naloxone. His oxygen saturation was noted to be $50 \%$ on room air, he was tachypneic with a respiratory rate of 22 , and his heart rate ranged from 89 to 104 beats per minute. His blood pressure remained within normal limits at $128 / 62$. His chest X-ray was notable for patchy bilateral perihilar infiltrates and the patient was intubated postoperatively. An EKG revealed normal sinus rhythm, and cardiac enzymes were negative. He was diagnosed with naloxone-induced non-cardiogenic pulmonary edema supported by the temporal relationship of the causal drug and no other identifiable cause of his clinical picture. He received furosemide and underwent diuresis while intubated, with subsequent improvement in his oxygen requirements. His vitals remained stable and he was extubated $6 \mathrm{~h}$ later. A Naranjo assessment score of 6 was obtained, indicating a probable relationship between the patient's symptoms and the suspect drug.
\end{abstract}

Nasheena Jiwa

sheena99@gmail.com

1 Department of Medicine, St. Mary's Hospital, Waterbury, CT, USA

2 Department of Pulmonary and Critical Care, Yale-Waterbury Hospital, Waterbury, CT, USA

\section{Key Points}

Naloxone-induced non-cardiogenic pulmonary edema is a rare but serious complication that should be recognized in the setting of opioid detoxification.

The potential of respiratory failure after naloxone therapy highlights the necessity for clinicians to cautiously treat narcotic-induced respiratory depression by securing an airway prior to the administration of naloxone.

It is recommended to use the lowest effective dose of naloxone necessary to elicit the desired response.

\section{Introduction}

Naloxone is an opiate antagonist, and is used for reversal of opioid effects. The adverse reactions of this drug include hypertension, ventricular arrhythmias, cardiac arrest, seizures, and rarely, pulmonary edema [10]. Naloxone-induced non-cardiogenic pulmonary edema (NCPE) has been scarcely reported in literature, and in order to recognize this complication, naloxone should be administered cautiously with closer observation of patients undergoing opioid detoxification [1]. We describe a case of pulmonary edema following naloxone administration in a patient without pre-existing heart disease. 


\section{Case Study}

A 22-year-old man presented for a right-shoulder open subacromial decompression and distal clavicle excision due to a right shoulder acromioclavicular joint degeneration resulting in nerve impingement. His past medical history was notable for obesity with a body mass index (BMI) of 31, mild obstructive sleep apnea (OSA) with an apnea-hypopnea index (AHI) of 5.0/h, pre-diabetes mellitus, and psoriatic arthritis for which he was on adalimumab subcutaneous weekly. The patient took no other regular medications, had no listed allergies, and had no prior history of surgeries. His right shoulder pain was refractory to a 1-week course of high-dose ibuprofen therapy and 3 months of physical therapy, and he was therefore scheduled for an elective procedure to relieve his symptoms. During the procedure, he was given fentanyl $50 \mu \mathrm{g} /$ $5 \mathrm{~mL}$ intravenously for anesthesia, bupivacaine $0.5 \%$ $(5 \mathrm{mg} / 5 \mathrm{~mL}$ ) intravenously for peripheral and sympathetic nerve blockade, and midazolam $1 \mathrm{mg}$ intravenously for sedation. The duration of the procedure from the time of anesthesia to the conclusion of the procedure was approximately $90 \mathrm{~min}$. The procedure was concluded without any complications; however, the patient developed post-operative fentanyl-induced respiratory depression with a respiratory rate of 12 breaths per minute, but he maintained his oxygen saturation of $99 \%$ on room air. $\mathrm{He}$ was given intravenous naloxone $0.2 \mathrm{mg}$ to reverse the effects of anesthesia, and this subsequently resulted in acute oxygen desaturation to $50 \%$ on room air, leading to a suspicion of non-cardiogenic flash pulmonary edema. The rest of his vital signs were notable for a respiratory rate that increased to 22 breaths per minute, his heart rate ranged from 89 to 104 beats per minute and his blood pressure was 126/80 during the incident. The patient was intubated for acute hypoxic respiratory failure. An arterial blood gas obtained while he was intubated on $60 \% \mathrm{FiO}_{2}$ showed a $\mathrm{pH}$ of $7.42, \mathrm{PCO}_{2}$ of $39 \mathrm{mmHg}, \mathrm{PO}_{2}$ of $88 \mathrm{mmHg}, \mathrm{HCO} 3 \mathrm{~A}$ of $25.6 \mathrm{mmol} / \mathrm{L}$ with an oxygen saturation of $98 \%$. His ventilator mode was pressure regulated volume control, with settings consisting of a tidal volume of $450 \mathrm{cc}$, a respiratory rate of 15 breaths per minute, an $\mathrm{FIO}_{2}$ of 0.6 and a positive end expiratory pressure of $5 \mathrm{cmH}_{2} \mathrm{O}$ (Figs. 1, 2).

On physical examination, he was noted to have bilateral crackles at the lung bases, and his right shoulder incision site remained clean, dry and intact. His chest X-ray was significant for patchy bilateral perihilar infiltrates. An electrocardiogram was obtained that revealed normal sinus rhythm, and cardiac enzymes were noted to be within normal limits. An echocardiogram was not obtained. The patient was young, with no significant history of cardiac heart disease, and no prior history of exertional dyspnea or

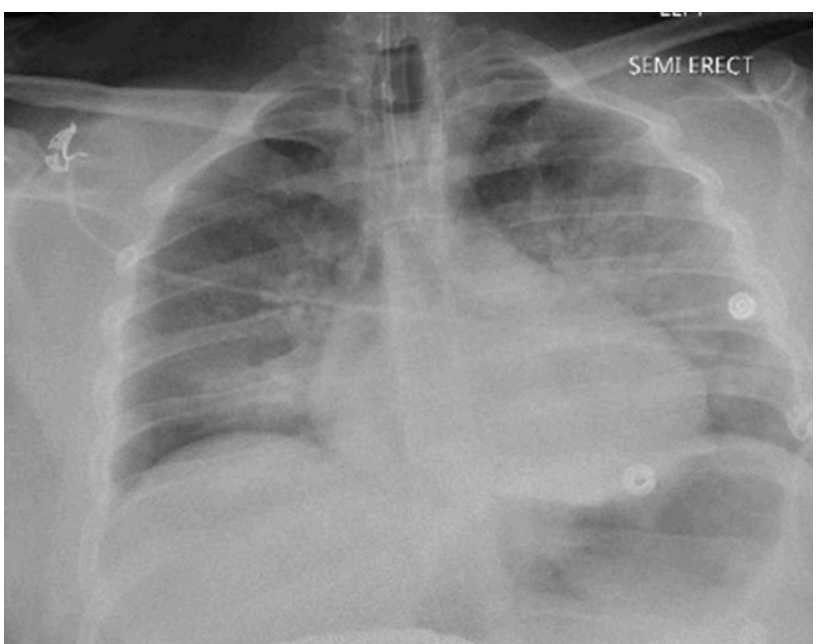

Fig. 1 Portable chest X-ray post intubation significant for patchy bilateral perihilar infiltrates, left greater than right

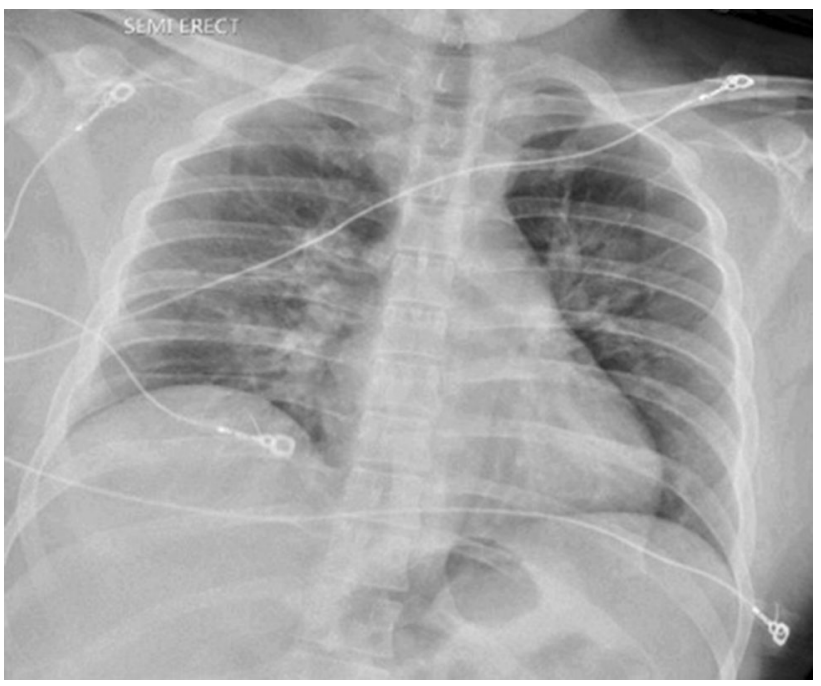

Fig. 2 Portable chest X-ray post extubation showing mild bilateral perihilar infiltrates representing resolving pulmonary edema since the prior examination

physical exam findings of a murmur, lower extremity edema, or jugular venous distension, giving heart failure a low probability as a cause of his clinical presentation with the preceding normal cardiac enzymes and electrocardiogram. He was given IV furosemide $40 \mathrm{mg}$, which resulted in approximately $3 \mathrm{~L}$ of urine output. He was maintained on mechanical ventilation for $6 \mathrm{~h}$ while he continued to diurese. He responded well with decreasing oxygen requirements and a successful spontaneous breathing trial after $6 \mathrm{~h}$ of intubation with continued diuresis. He was extubated to nasal cannula and monitored overnight in the intensive care unit. A repeat chest X-ray after extubation showed resolving bilateral pulmonary edema correlating with clinical improvement. 
The suspicion that naloxone was the cause of the adverse event of pulmonary edema was based on several factors, resulting in a Naranjo score of 6 . The adverse drug reaction occurred after the suspected drug was given, and other causes of flash pulmonary edema were considered and investigated. The patient had no history, symptoms, or physical exam findings of heart failure or uncontrolled hypertension, and his concurrent OSA and obesity were both mild in nature, making these factors unlikely as a cause of flash pulmonary edema independent of a triggering event. This, in addition to prior reports of naloxoneinduced non-cardiogenic pulmonary edema, yields a Naranjo score of 6 , indicating a probable relationship between the patient's symptoms and the suspect drug. The patient was discharged with no long-term sequelae or complications from his procedure or subsequent medication effect.

\section{Discussion}

Naloxone is a competitive $\mu$-opioid-receptor antagonist that reverses the effects of opioid intoxication [2]. Naloxone-induced NCPE is suggested to occur at a rate ranging from 0.2 to $3.6 \%$ in patients [13]. The pulmonary edema is likely caused by an adrenergic response from a large increase in centrally mediated catecholamines following naloxone administration [14]. When naloxone is given to a patient, a catecholamine-mediated response is elicited [4]. The effect of catecholamines results in hypertension, tachycardia, and diaphoresis. This was demonstrated by the patient's increase in heart rate and respiratory rate. The patient's blood pressure remained within the same normotensive range during the incident. The patient's hypoxia occurred after the administration of naloxone; therefore, there was no evidence of hypoxia prior to the administration of naloxone or during the event of respiratory depression as a cause of the patient's clinical picture of pulmonary edema. It is probable that our patient developed signs of a catecholamine surge such as tachycardia and tachypnea, which is likely due to the pathophysiologic role of naloxone in causing pulmonary capillary permeability [3]. The subsequent sympathetic release of epinephrine in plasma antagonizes the $\mu$-opioid receptors in the adrenal medulla. The increase in sympathetic outflow causes a shift in blood volume into the pulmonary vasculature, causing subsequent pulmonary vasoconstriction and pulmonary hypertension [1].

Approximately $2-11 \%$ of patients with OSA may be susceptible to postoperative respiratory complications requiring naloxone for opioid detoxification [6], as demonstrated by our patient. Although the patient has a concurrent history of mild OSA and obesity, which may contribute to his post-operative risk of respiratory depression, these factors may be independent of his likelihood of developing pulmonary edema. Our patient did not have evidence of significant airway obstruction with an AHI of 5, therefore the incident of pulmonary edema was less likely to have occurred independent of a triggering event, given that patients with pre-existing heart disease or young healthy patients can have this complication occur with standard dosages of naloxone ranging from 0.2 to $0.4 \mathrm{mg}$, or even with smaller dosages as low as 0.04-0.08 mg [6]. Our patient was a young and healthy individual without a history of heart disease, and no other cause of pulmonary edema or hypoxemia was identified; therefore, his clinical picture was most consistent with the diagnosis of naloxone-induced acute pulmonary edema, which was further supported by the temporal relationship of the causal medication. The patient's short course of ibuprofen therapy was unlikely to contribute to his clinical presentation of pulmonary edema following naloxone administration given his normal renal function and absence of cardiac heart disease. In addition, the patient was not on any other medication that could be identified as contributing to his clinical presentation.

Several reports suggest airway obstruction with the administration of fentanyl. In a recent a case report by Khan et al., fentanyl was administered in a patient who demonstrated acute hypoxic respiratory failure, wheezing, and respiratory distress several minutes after the administration of the drug [7]. Additionally, Phua et al. suggest that fentanyl-induced chest wall rigidity peaks $3 \mathrm{~min}$ after the administration of fentanyl and can last for $11.5 \mathrm{~min}$ [11]. Although our patient received fentanyl at the initiation of the procedure, and was noted to have respiratory depression, he did not experience respiratory distress, or hypoxic respiratory failure until after the administration of naloxone at the conclusion of the 90-min procedure; therefore, fentanyl was unlikely the cause of our patient's respiratory distress.

The management of non-cardiogenic edema in the following several reports suggests the administration of diuretics. In Schwartz and Koenigsberg, the administration of furosemide and nitroglycerine morphine sulfate resulted in the resolution of naloxone-induced non-cardiogenic edema [12]. Furthermore, Lynn and Galinkin suggest that the pulmonary edema resulting from naloxone resolves quickly in response to a diuretic or even, in some cases, additional opioid [9].

Similar cases have been described in various case reports. In Bansal et al., the patient developed acute respiratory distress after receiving naloxone for an opioid overdose [1]. Similarly, the patient was treated with intravenous furosemide and extubated $8 \mathrm{~h}$ later with resolving pulmonary infiltrates. The patient described in the case report by Horng et al. had a history of OSA; 
however, the severity of this was not specified [6]. The patient in this case also developed post-operative fentanylinduced respiratory depression and received naloxone $0.08 \mathrm{mg}$, which subsequently resulted in acute pulmonary edema in the setting of upper airway obstruction in addition to naloxone administration. Our patient had a similar presentation given that the patient was noted to have respiratory depression in the setting of mild OSA, however no significant airway obstruction was noted. Our patient also rapidly improved with IV furosemide and short-term intubation.

Although the use of naloxone to reverse respiratory depression provides advantages such as eliminating the need for postoperative mechanical ventilation, potential consequences also need to be considered [5]. Because of this rare but potential complication, a secure airway should be obtained before naloxone administration for the treatment of narcotic-induced respiratory depression. This case highlights a critical incident of respiratory failure after naloxone administration. Pulmonary edema is a known but very rare side effect of naloxone and naloxone-containing medicinal products. Other potential deleterious effects include possible precipitation of severe pain, acute drug withdrawal, and circulatory consequences, especially in cardiac patients [5].

Given that pulmonary edema can cause significant hypoxemia and cardiac instability refractory to oxygen supplementation when associated with inability to protect the airway in the setting of opioid use, naloxone is still considered the initial therapy to restore and maintain the airway for ventilation in a patient with opioid-induced respiratory symptoms [8]. Although it is unclear if the occurrence of adverse symptoms such as pulmonary edema is dose dependent, newer guidelines from the American Heart Association recommend using the lowest effective dose of naloxone necessary to elicit the desired response [9].

\section{Compliance with ethical standards}

Consent Written informed consent was obtained from the patient for publication of this case report and any accompanying images. A copy of the written consent may be requested for review from the corresponding author.

Disclosures No financial support was received for the conduct of this study or preparation of this manuscript.

Conflict of interest Nasheena Jiwa, Himesh Sheth, and Richard Silverman declare that they have no conflict of interest.
Open Access This article is distributed under the terms of the Creative Commons Attribution-NonCommercial 4.0 International License (http://creativecommons.org/licenses/by-nc/4.0/), which permits any noncommercial use, distribution, and reproduction in any medium, provided you give appropriate credit to the original author(s) and the source, provide a link to the Creative Commons license, and indicate if changes were made.

\section{References}

1. Bansal S, Khan R, Tietjen P. Naloxone induced pulmonary edema. Chest. 2007;132:692.

2. Boyer EW. Management of opioid analgesic overdose. N Engl J Med. 2012;367:146-55. https://doi.org/10.1056/ NEJMra1202561.

3. Congeni A, Fox A, King A. The case files: serious and unusual reaction to nalxone administration. Emerg Med News. 2015;37(7A). https://doi.org/10.1097/01.eem.0000469915.22317. $8 \mathrm{~d}$.

4. Dahan A, Aarts L, Smith TW. Incidence, reversal, and prevention of opioid-induced respiratory depression. J Am Soc Anesthesiol. 2010;112(1):226-38.

5. Flacke JW, Flacke WE, Williams GD. Acute pulmonary edema following naloxone reversal of high dose morphine anesthesia. J Am Soc Anesthesiol. 1977;47:376-8.

6. Horng HC, Ho MT, Huang CH, Yeh CC, Cherng CH. Negative pressure pulmonary edema following naloxone administration in a patient with fentanyl induced respiratory depression. Acta Anaesthesiol Taiwan. 2010;48(3):155-7.

7. Khan M, Agarwal A, Abouzgheib W. Fentanyl induced chest wall rigidity and negative pressure pulmonary edema. Chest. 2015;148:1025A. https://doi.org/10.1378/chest.2271026.

8. Lavonas EJ, Drennan IR, Gabrielli A, et al. Part 10: special circumstances of resuscitation: 2015 American Heart Association guidelines update for cardiopulmonary resuscitation and emergency cardiovascular care. Circulation. 2015;132(Suppl. 2):S501-18.

9. Lynn RR, Galinkin JL. Naloxone dosage for opioid reversal: current evidence and clinical implications. Adv Drug Saf. 2018;9(1):63-8. https://doi.org/10.1177/2042098617744161.

10. Partridge BL, Phil D, Ward CF. Pulmonary edema following lowdose naloxone administration. J Am Soc Anesthesiol. 1986;65(6):709-10.

11. Phua CK, Wee A, Lim A, Abishegenaden J, Verma A. Fentanylinduced chest wall rigidity syndrome in a routine bronchoscopy. Respir Med Case Rep. 2017;20:205-7. https://doi.org/10.1016/j. rmcr.2017.02.012.

12. Schwartz JA, Koenigsberg MD. Naloxone-induced pulmonary edema. Ann Emerg Med. 1987;16(11):1294-6.

13. Sporer KA, Firestone J, Isaacs SM. Out of hospital treatment of opioid overdoses in an urban setting. Acad Emerg Med. 1996;3(7):660-7.

14. Taff RH. Pulmonary edema following naloxone administration in a patient without heart disease. J Am Soc Anesthesiol. 1983;59(6):576-7. 